\title{
10 \\ ISSN: $2220-4822$ \\ Phytochemical analysis of Gloriosa superba L. using GC-MS from five different ecotypes of Tamil Nadu State, India
}

\author{
J.A. Paul Jasmine ${ }^{1}$, T. Sundari, ${ }^{2}$ V. Balakrishnan ${ }^{1,3 *}$ \\ 'Research and Development Center, Bharathiar University, Coimbatore-641 046, Tamil Nadu, India, ${ }^{2}$ Department \\ of Chemistry, K.S. Rangasamy College of Engineering, Tiruchengode-637 215 Tamil Nadu, India, ${ }^{3} P G$ and Research \\ Department of Botany, Arignar Anna Government Arts College, Sanyasikaradu, Namakkal - 637 002, Tamil Nadu, \\ India
}

Received: September 21, 2019 Revised: January 15, 2020 Accepted: January 18,2020 Published: February 08, 2020

\section{*Corresponding Author:}

V. Balakrishnan

Email: palanivbalu@gmail.com

\begin{abstract}
Gloriosa superba $\mathrm{L}$ is an important medicinal plant and its seeds, tubers are used for medicine. To investigate the phyto-components of Gloriosa superba L were collected from various habitats of Tamil Nadu state, India. In the present study, the phyto-components from the tubers of Gloriosa superba L cultivars from Sirumalai (GAl) Mulanoor (GA2), Thuraiyur (GA3), Konganapuram (GA4) and Vedaranyan (GA5) were extracted with ethanol and the composition of chemicals and its concentration in the tubers were determined by Gas Chromatography - Mass spectrometry (GC-MS) analysis.Among the phyto-components GAl showed 15 phyto-components, GA2 showed 13 phyto-components, GA3 showed 8 phyto-components, GA4 showed 14 phyto-components and GA5 showed 13 phyto-components. GA1, GA2, GA4 and GA5 ecotypes possessed higher phyto-components. Colchichine an important alkaloid of Gloriosa superba L was found in GA2, GA3, GA4 and GA5 accessions in good concentration. The results reveals that the geographical origin and climatic condition of a accession caused polymorphisms in the accumulation of phyto-components, its composition and morphological traits in Gloriosa Superba L originating from different ecotypes of Tamil Nadu state.
\end{abstract}

KEYWORDS: Accessions, cultivars, GC-MS, Gloriosa superba L, habitat, phyto-components

\section{INTRODUCTION}

Gloriosa Superba L. belongs to the family Colchicaceae. This plant is popularly known by other terminologies such as "Glory Lily", Kalihari, Ognisikha etc., due to the presence of wavy edged yellow and red flowers. G. superba is a perennial climbing glabrous herb with tuberous root. All the parts of the plant especially tuber is poisonous. The plant species contain high amount of Colchicine, which is a toxic alkaloid. It also contains alkaloid Gloriocine. The seeds of Gloriosa superba L is an important source for Colchicine extraction. The plant almost distributed throughout India and also found in forests of Tamil Nadu.

In some Districts, farmers cultivate the plant species for its seeds. Tuber is a rich source of alkaloid colchicine [1]. Most of the medicinal plants were the best source to get a variety of new herbal products [2]. In current scenario WHO estimated
$80 \%$ of the people from all over the world are interested towards traditional medicine. Medicinal plants are commonly known as people's friend, providing the food, fuel and medicine [3]. Medicinal plant resources are major sources of Indian traditional and modern medicine [4\&5].

The tubers are commonly used for the treatment of inflammation, ulcers, bleeding piles, white discharge, scrofula, skin diseases, leprosy, indigestion, helminthes, snake bites, intermittent fever, baldness and debility [6\&7]. Most of the research findings are plant oriented products towards identifying the chemical compounds especially secondary metabolites [8].

Mostly medicinal plants are warrantly being screened for their biological and pharmacological activities such as anti-diabetic, antioxidant, antimicrobial, laxative and anti cancer activities [9-14] . India was sourced with its rich traditional knowledge and has heritage of herbal products and medicines and enhanced its

Copyright: $\odot$ The authors. This article is open access and licensed under the terms of the Creative Commons Attribution License (http://creativecommons.org/licenses/by/4.0/) which permits unrestricted, use, distribution and reproduction in any medium, or format for any purpose, even commercially provided the work is properly cited. Attribution - You must give appropriate credit, provide a link to the license, and indicate if changes were made. 
richness with varying biodiversity [15]. The present investigation was carried out to evident the presence of phyto-components with varying quality and quantity in the samples of Gloriosa superba L collected from five different accessions of Tamil Nadu State, India.

\section{MATERIALS AND METHODS}

\section{Plant Collection}

The five ecotypes of Gloriosa superba $\mathrm{L}$ cultivated in the places such as Sirumalai (GA1), Mulanoor (GA2), Thuraiyur (GA3), Konganapuram (GA4) and Vedaranyam (GA5) belongs to the districts such as Dindigul, Tiruppur, Trichy, Salem and Nagapatinam respectively (Figure 1). They were indentified and authenticated by the Botanical Survey of India, (Southern Circle), Coimbatore, Tamil Nadu, India. The plants were deposited in the department of Botany, Arignar Anna Government Arts College, Namakkal, Tamil Nadu, India.

\section{Geographical Location of Study Area}

Sirumalai (GAl) is a region of 60,000 acres $(200 \mathrm{~km} 2)$ situated $25 \mathrm{~km}$ from Dindigul town. The latitude is $10^{\circ} 11^{\prime} 39.28^{\prime \prime} \mathrm{N}$ and longitude is 77059'48.14"E. Elevation is 1092.63 Meters (3584.75 Feet). Mulanoor (GA2) is located in Tiruppur district. Mulanur is located at $10.77^{\circ} \mathrm{N} 77.72^{\circ} \mathrm{E}$. It has an average elevation of 238 metres (780 feet).Mulanoor is a part of Glory lily market.

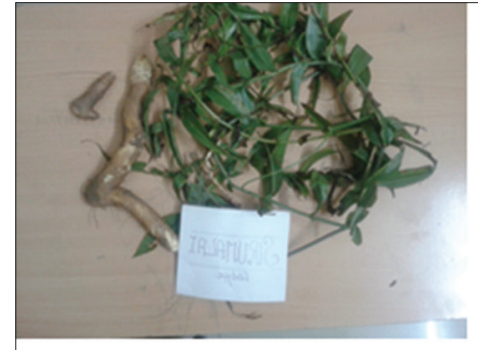

Sirumalai (GA1)

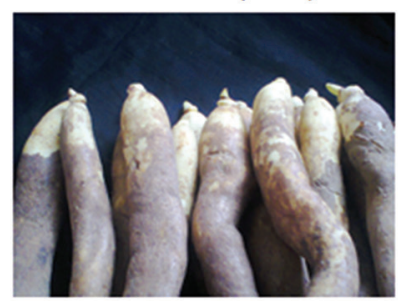

Thuraiyur (GA3)

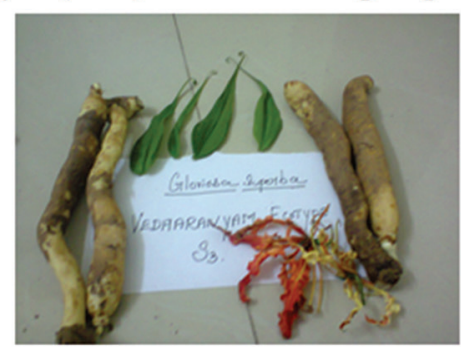

Vedaranyam (GA5)

Figure 1: Morphological view of different type of ecotypes
Thuraiyur (GA3) latitude is $11^{\circ} 8$ ' 29.2380" $\mathrm{N}$ and longitude is $78^{\circ} 35^{\prime} 40.1100^{\prime \prime}$ E. Situated in Tiruchirappalli district. Konganapuram (GA4) is located at $11.58^{\circ} \mathrm{N} 77.92^{\circ} \mathrm{E}$.It has an average elevation of 300 metres (1000 feet).Situated in Salem district. Vedaranyam (GA5) belongs to Nagapattinam district. The latitude is $10^{\circ} 22^{\prime 2} 27.15^{\prime \prime} \mathrm{N}$ and longitude is $79^{\circ} 51^{\prime} 27.66^{\prime \prime} \mathrm{E}$. The elevation is 2.36 Meters (7.73 Feet).

\section{Preparation of Powder and Extract}

One kilogram of tubers was shade dried and powdered. The powder extracted with ethanol for 8 hours by using Soxhlet apparatus. After 8 hours, the extract was filtered through muslin cloth, evaporated under reduced pressure condition and vacuum dried to get the viscous residue. Ethanolic extract of Gloriosa superba L was used for GC-MS analysis. $2 \mu \mathrm{l}$ of the ethanolic leaf extract of Gloriosa superba L employed for GC-MS. Yield of extract was calculated by yield $(\mathrm{g} / 100 \mathrm{~g})=(\mathrm{W} 1 \mathrm{x} 100) / \mathrm{W} 2$, where $\mathrm{Wl}$ is the weight of the extract residue obtained after solvent removal and W2 is the weight of tuber taken.

\section{GC-MS Analysis}

GC-MS analysis on the ethanolic extract of Gloriosa superba $\mathrm{L}$ was carried out in the Indian Institute for Crop Processing Technology (IICPT), Thanjavur. GC clarus 500 perkin Elmer system comprising a Aoc - 20i auto sampler and gas chromatography interfaced to a pass petometer (GC- MS) instrument was used, applying following conditions [16].

\section{Column}

Elite- 1 silica capillary column $(30 \mathrm{~mm} \times 0.25 \mathrm{~mm}$ ID $\times \mathrm{M} \mu \mathrm{M}$ df composed of $100 \%$ Dimethyl poly siloxane), operating in electron impact mode at $70 \mathrm{ev}$ was used.

\section{Carrier Gas}

Helium (99.99\%) generally used as carrier gas at a constant flow of $1 \mathrm{ml} / \mathrm{min}$ and an injection volume of $0.5 \mu \mathrm{l}$ was employed (split ratio of $10: 1$ ) at injector temperature $250^{\circ} \mathrm{C}$ and ion source temperature $280^{\circ} \mathrm{C}$. The oven temperature generally programmed from $110^{\circ} \mathrm{C}$, with an increase of $10^{\circ} \mathrm{C} / \mathrm{min}$, to $200^{\circ} \mathrm{C}$, then $5^{\circ} \mathrm{C} / \mathrm{min}$ up to $280^{\circ} \mathrm{C}$, finally ending with a $9 \mathrm{~min}$ isothermal at $280^{\circ} \mathrm{C}$.

\section{Spectra}

Mass spectra were taken at $70 \mathrm{eV}$ with a scan interval of 0.5 seconds and fragments from 45 to $450 \mathrm{Da}$. GC total running time is 36 minutes. The plant extract was dissolved in methanol and filtered with polymeric solid phase extraction (SPE) column and analyzed in for different components.

\section{Component Identification}

Here interpretation on mass spectrum GC-MS was conducted by using database of National Standard and Technology (NIST) 
library, having more than 62,000 patterns. The spectrum of unknown component was compared with the spectrum

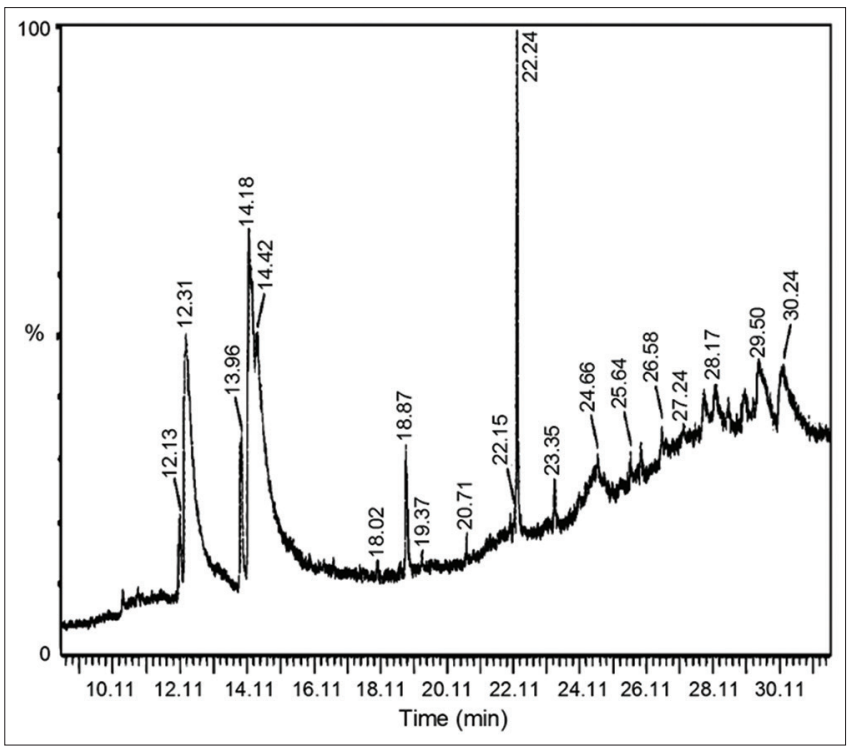

Figure 2: GC-MS chromatogram of tuber methanol extract of Gloriosa superba L (GA1 - Sirumalai) ecotype of the known components stored in the NIST library [17] The compound name, molecular weight and structure of the components for the test materials were ascertained.

\section{RESULTS}

The GC-MS analysis is the valuable method which has been increasingly applied for the analysis of medicinal plants for nonpolar components, volatile essential oil, fatty acids and alkaloids. The Gloriosa superba L of Sirumalai accession (GAl) subjected to GC-MS analysis retrieved 15 major phyto-components.

Such as Deconoic acid, Ethyl ester, n-Hexadecanoic acid, Oleyl Alohol, 9,12- Octadecadienoic acid (Z, Z), Dodecanoic acid, 2- Penten -1-yl ester, 1,2 - Benzenedicarboxylic acid, Diisoctyl ester, 1 - Octadecyne, Squalene, 1 - Eicosanol, Pentanoic acid, 10 - Undecenyl ester, 9-Octadecenoic acid (Z) - Phenylmethyl ester, 6,9,12, Octadecatrienoic acid, Phenyl methyl ester (Z,Z,Z), [1, l' Bicycloprophyl]-2-Octanoic acid, 2'-hexyl-, methyl ester, 9,12-Octadecadienoic (Z Z)- Phenylmethyl ester (Table 1 and Figure 2).

Gloriosa superba L cultivated in Mulanoor region(GA2) of Tiruppur district. This ecotypes contained thirteen different

Table 1: Phyto-components obtained through GC-MS analysis isolated from methanolic tuber extracts of Gloriosa superba L (GAl-Sirumalai) ecotype

\begin{tabular}{|c|c|c|c|c|c|}
\hline S.No. & RT & Name of the compound & Molecular formulae & MW & Peak area \% \\
\hline 1. & 12.13 & Decanoic acid, Ethyl ester & $\mathrm{C}_{12} \mathrm{H}_{24} \mathrm{O}_{2}$ & 200 & 1.78 \\
\hline 2. & 12.31 & n-Hexadecanoic acid & $\mathrm{C}_{16} \mathrm{H}_{32} \mathrm{O}_{2}$ & 256 & 24.34 \\
\hline 3. & 13.96 & Oleyl Alcohol & $\mathrm{C}_{18} \mathrm{H}_{36} \mathrm{O}$ & 268 & 1.94 \\
\hline 4. & 14.18 & $9,12-0$ ctadecadienoic acid $(Z, Z)$ - & $\mathrm{C}_{18} \mathrm{H}_{32} \mathrm{O}_{2}$ & 280 & 15.57 \\
\hline 5. & 18.02 & Dodecanoic acid,2-penten-1-yl ester & $\mathrm{C}_{17} \mathrm{H}_{32} \mathrm{O}_{2}$ & 268 & 0.22 \\
\hline 6. & 18.87 & 1,2-Benzenedicarboxylic acid, diisooctyl ester & $\mathrm{C}_{24} \mathrm{H}_{38} \mathrm{O}_{4}$ & 390 & 2.45 \\
\hline 7. & 20.71 & 1-0ctadecyne & $\mathrm{C}_{18}^{24} \mathrm{H}_{34}$ & 250 & 0.36 \\
\hline 8. & 22.24 & Squalene & $\mathrm{C}_{30} \mathrm{H}_{5}$ & 410 & 6.52 \\
\hline 9. & 23.35 & 1-Eicosanol & $\mathrm{C}_{20} \mathrm{H}_{42} \mathrm{O}$ & 298 & 0.63 \\
\hline 10. & 24.66 & Pentanoic acid, 10-undecenyl ester & $\mathrm{C}_{16} \mathrm{H}_{30} \mathrm{O}_{2}$ & 254 & 1.68 \\
\hline 11. & 25.95 & 1-Octadecyne & $\mathrm{C}_{18} \mathrm{H}_{3}$ & 250 & 0.85 \\
\hline 12. & 27.24 & 9-Octadecenoic acid (Z)-,Phenylmethyl ester & $\mathrm{C}_{25} \mathrm{H}_{40} \mathrm{O}_{2}$ & 372 & 3.69 \\
\hline 13. & 28.17 & $6,9,12-0$ ctadecatrienoic acid,phenylmethyl ester, $(Z, Z, Z)$ - & $\mathrm{C}_{25} \mathrm{H}_{36} \mathrm{O}_{2}$ & 368 & 9.33 \\
\hline 14. & 29.50 & {$\left[1,1^{\prime}\right.$-Bicyclopropyl]-2-octanoic acid, $2^{\prime}$-hexyl-, methyl ester } & $\mathrm{C}_{21} \mathrm{H}_{38} \mathrm{O}_{2}$ & 322 & 14.39 \\
\hline 15. & 30.24 & 9,12-0ctadecadienoic $(Z, Z)$-,phenylmethyl ester & $\mathrm{C}_{25} \mathrm{H}_{38} \mathrm{O}_{2}$ & 370 & 16.23 \\
\hline
\end{tabular}

Table 2 : Phyto-components obtained through GC-MS analyzing isolated from methanolic tuber extracts of Gloriosa superba $\mathrm{L}$ (GA2-Mulanoor) ecotype

\begin{tabular}{|c|c|c|c|c|c|}
\hline S.No. & RT & Name of the compound & Molecular formulae & MW & Peak area \% \\
\hline 1. & 4.03 & 2,3-Dimethyl fumaric acid & $\mathrm{C}_{6} \mathrm{H}$ & 144 & 3.82 \\
\hline 2. & 5.78 & Salicyl Alcohol & $\mathrm{C}_{7}^{6} \mathrm{H}_{8}^{8} \mathrm{O}_{2}^{4}$ & 124 & 10.29 \\
\hline 3. & 6.73 & 2-[3-Hydroxy-4-methoxyphenyl]-semicarbazide & $\mathrm{C}_{8} \mathrm{H}_{11} \mathrm{~N}_{3} \mathrm{O}_{3}$ & 197 & 2.70 \\
\hline 4. & 8.41 & Benzoic acid,2-hydroxy-6-methoxy & $\mathrm{C}_{8} \mathrm{H}_{8} \mathrm{O}_{4}$ & 168 & 9.08 \\
\hline 5. & 11.06 & Undecanoic acid & $\mathrm{C}_{11} \mathrm{H}_{22} \mathrm{O}_{2}$ & 186 & 1.26 \\
\hline 6. & 12.14 & n-Hexadecanoic acid & $\mathrm{C}_{16}^{11} \mathrm{H}_{32} \mathrm{O}_{2}$ & 256 & 24.36 \\
\hline 7. & 13.81 & 9,12-0ctadecadienoic acid,methyl ester, $(E, E)$ - & $\mathrm{C}_{19} \mathrm{H}_{34} \mathrm{O}_{2}$ & 294 & 2.00 \\
\hline 8. & 14.17 & 9,12-0ctadecadienoic acid $(Z, Z)-$ & $\mathrm{C}_{18} \mathrm{H}_{32} \mathrm{O}_{2}$ & 280 & 32.09 \\
\hline 9. & 17.95 & 2-Hydroxy-(Z)9-pentadecenyl propanoate & $\mathrm{C}_{18} \mathrm{H}_{34} \mathrm{O}_{3}$ & 298 & 0.23 \\
\hline 10. & 18.72 & 10-Undecenoic acid, octyl ester & $\mathrm{C}_{19} \mathrm{H}_{36} \mathrm{O}_{2}$ & 296 & 0.35 \\
\hline 11. & 27.91 & Cholestan-3-ol,2-Methylene-,(3a,5a)- & $\mathrm{C}_{28} \mathrm{H}_{48} \mathrm{O}$ & 400 & 0.96 \\
\hline 12. & 29.40 & Lumicolchicine & $\mathrm{C}_{22} \mathrm{H}_{25} \mathrm{O}_{6}$ & 399 & 10.04 \\
\hline 13. & 30.00 & Ethyl iso-allocholate & $\mathrm{C}_{26} \mathrm{H}_{44} \mathrm{O}_{5}$ & 436 & 2.82 \\
\hline
\end{tabular}


compounds. The compounds such as 2,3-Dimethyl fumaric acid, Salicyl Alcohol, 2-[3-Hydroxy-4-methoxyphenyl]semicarbazide, Benzoic acid,2-hydroxy-6-methoxy, Undecanoid acid, n-Hexadecanic acid, 9, 12-Octadecadienoic acid,methyl ester,(E,E)-, 9,12-Octadecadienoic acid (Z,Z)-, 2-Hydroxy-(Z)9-pentadecenyl propanoate, 10- Undecenoic acid, Octyl ester, Cholestan-3-ol,2-Methylene-,(3a,5a)-, Lumicolchicine, Ethyl iso-allocholate were identified (Table 2 and Figure 3).

Gloriosa superba L the ecotypes from Thuraiyur (GA3), Trichy district recorded eight compounds such as Lactose, Undecanoic acid, n-Hexadecanoic acid, 9,12- Octadecadienoic acid (Z, Z), 2-Hydroxy - (Z) 9-Pendadecenyl propanoate, 10- Undecenoic acid, Octyl

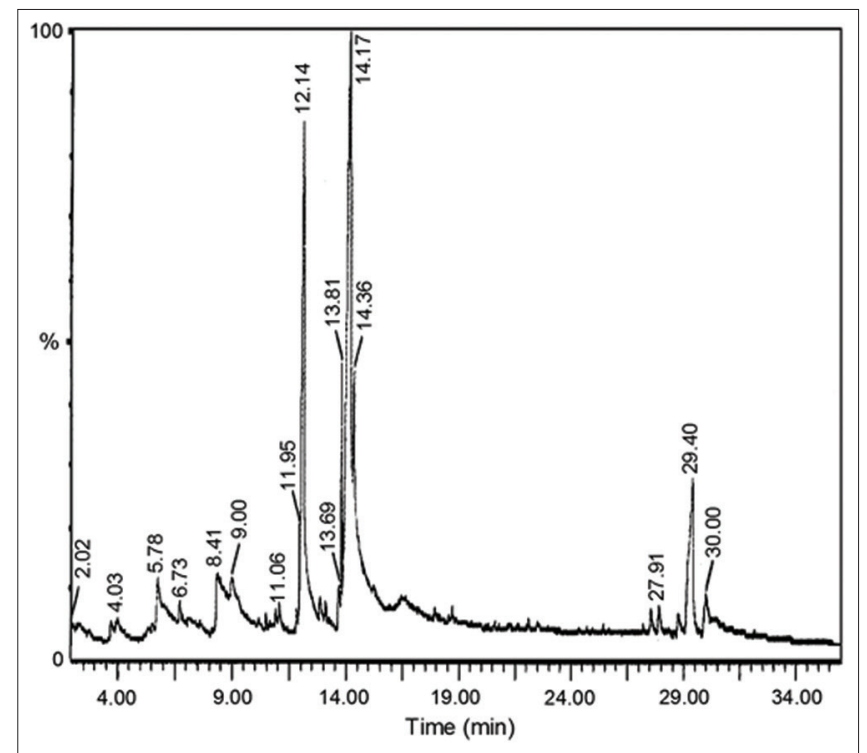

Figure 3: GC-MS chromatogram of tuber methanol extract of Gloriosa superba L (GA2 - Mulanoor) ecotype

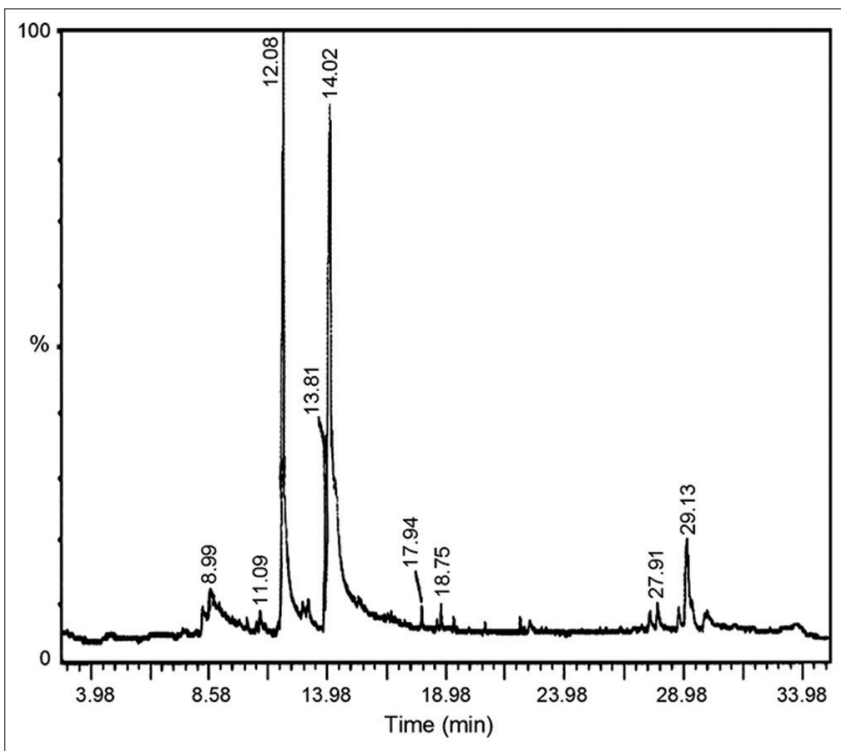

Figure 4: GC-MS chromatogram of tuber methanol extract of Gloriosa superba L (GA3 - Thuraiyur) ecotype ester, Cholestan -3-ol, 2-Methylene,(3a, 5a)-, Lumicolchicine (Table 3 and Figure 4).

There were thirteen chemical compounds recorded in GA4. They are Lactose, Benzene 1,4-bis(1,1-dimethyl ether), n-Hexadecanoid acid, 9,12- Octadeca dienoic acid (Z, Z), 10-Methyl-E-11-Tridecen-1-ol propionate, 2-Hydroxy - (Z) 9-Pendadecenyl propanoate, 10- Undecenoic acid, Octyl ester, Squalene, Cis-Z-a-Bisabolene epoxide, Cholestan-3-ol, 2-Methylene,(3à, 5à)- Lumicolchicine, Vitamin A aldehyde, Lumicolchicine and Ethyl iso-allocholate (Table 4 and Figure 5).

There are thirteen chemical compounds are identified in the ecotype of Vedaranyam (GA5) Gloriosa superba L. The chemical compounds such as lactose, Undecanoic acid, n-Hexadecanoic

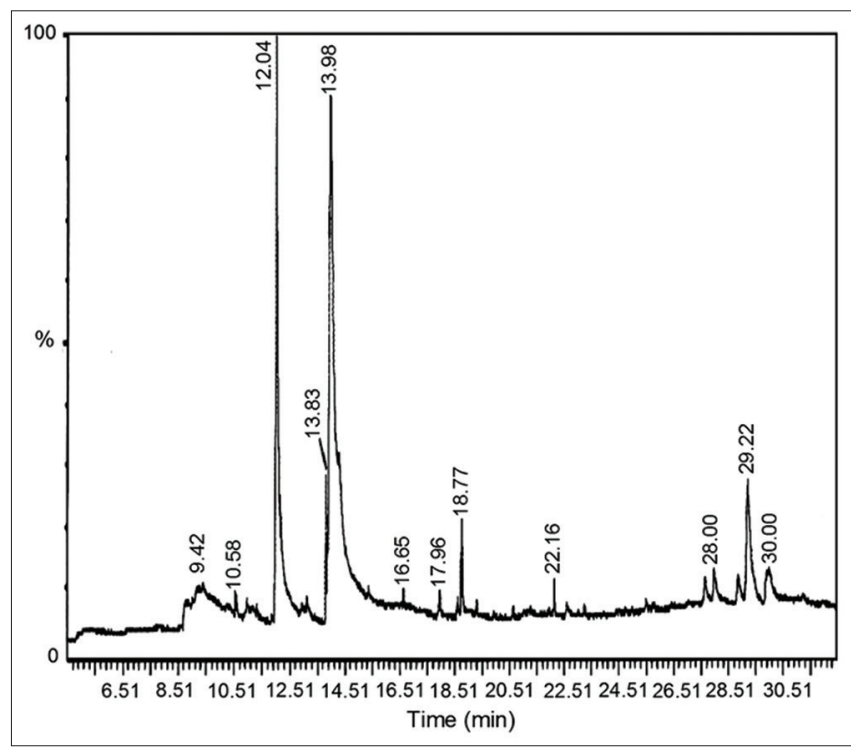

Figure 5: GC-MS chromatogram of tuber methanol extract of Gloriosa superba L(GA4 - Konganapuram) ecotype

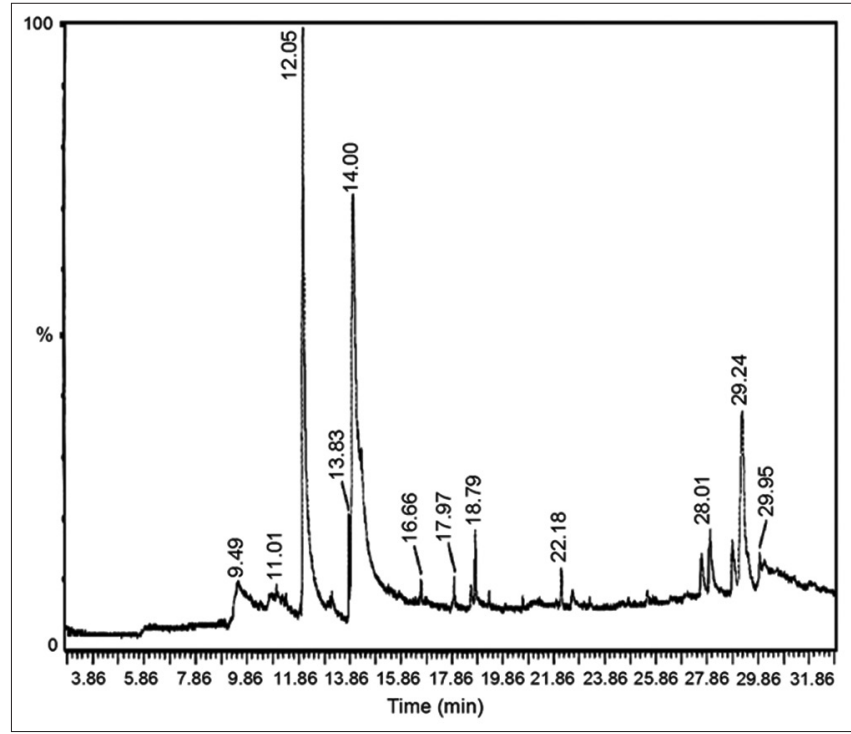

Figure 6: GC-MS chromatogram of tuber methanol extract of Gloriosa superba L (GA5 - Vedaranyam) ecotype 
Table 3: Phyto-components obtained through GC-MS analysis isolated from methanolic tuber extracts of Gloriosa superba $\mathrm{L}$ (GA3-Thuraiyur) ecotype

\begin{tabular}{|c|c|c|c|c|c|}
\hline S.No. & RT & Name of the compound & Molecular formulae & $\mathrm{MW}$ & Peak area \% \\
\hline 1. & 8.99 & Lactose & $\mathrm{C}_{12} \mathrm{H}_{22} \mathrm{O}_{11}$ & 342 & 5.29 \\
\hline 2. & 11.09 & Undecanoic acid & $\mathrm{C}_{11}^{12} \mathrm{H}_{22}^{22} \mathrm{O}_{2}$ & 186 & 0.83 \\
\hline 3. & 12.08 & n-Hexadecanoic acid & $\mathrm{C}_{16}^{11} \mathrm{H}_{32} \mathrm{O}_{2}^{2}$ & 256 & 32.77 \\
\hline 4. & 14.02 & 9,12-0ctadecanoic acid $(Z, Z)-$ & $\mathrm{C}_{18}^{16} \mathrm{H}_{32}^{32} \mathrm{O}_{2}^{2}$ & 280 & 53.30 \\
\hline 5. & 17.94 & 2-Hydroxy-(Z) 9-pentadecenyl propanoate & $\mathrm{C}_{18}^{18} \mathrm{H}_{34}^{32} \mathrm{O}_{3}^{2}$ & 298 & 0.41 \\
\hline 6. & 18.75 & 10- Undecenoic acid,octyl ester & $\mathrm{C}_{19}^{18} \mathrm{H}_{36}^{34} \mathrm{O}_{2}^{3}$ & 296 & 0.47 \\
\hline 7. & 27.91 & Cholestan-3-ol,2-methylene-, $(3 a, 5 a)-$ & $\mathrm{C}_{28}^{19} \mathrm{H}_{48}^{36} \mathrm{O}^{2}$ & 400 & 1.16 \\
\hline 8. & 29.13 & Lumicolchicine & $\mathrm{C}_{22}^{28} \mathrm{H}_{25}^{48} \mathrm{NO}_{6}$ & 399 & 5.78 \\
\hline
\end{tabular}

Table 4: Phyto-components obtained through GC-MS analyzing isolated from methanolic tuber extracts of Gloriosa superba $\mathrm{L}$ (GA4-Konganapuram) ecotype

\begin{tabular}{|c|c|c|c|c|c|}
\hline S.No. & RT & Name of the compound & Molecular formulae & MW & Peak area $\%$ \\
\hline 1. & 9.42 & Lactose & $\mathrm{C}_{12} \mathrm{H}_{22} \mathrm{O}_{11}$ & 342 & 6.06 \\
\hline 2. & 10.58 & Benzene,1,4-bis (1,1-dimethyl ether)- & $\mathrm{C}_{14}^{12} \mathrm{H}_{22}^{22}{ }^{11}$ & 190 & 1.11 \\
\hline 3. & 12.04 & $\mathrm{n}$-Hexadecanoic acid & $\mathrm{C}_{16}^{14} \mathrm{H}_{32}^{22} \mathrm{O}_{2}$ & 256 & 22.80 \\
\hline 4. & 13.98 & 9,12-0ctadecadienoic acid $(\mathrm{Z}, \mathrm{Z})$ - & $\mathrm{C}_{18}^{16} \mathrm{H}_{32}^{32} \mathrm{O}_{2}^{2}$ & 280 & 39.64 \\
\hline 5. & 16.65 & 10-Methyl-E-11-tridecen-1-ol propionate & $\mathrm{C}_{17}^{18} \mathrm{H}_{32}^{32} \mathrm{O}_{2}^{2}$ & 268 & 0.29 \\
\hline 6. & 17.96 & 2-Hydroxy-(Z)9-pentadecenyl propanoate & $\mathrm{C}_{18}^{1 /} \mathrm{H}_{34}^{32} \mathrm{O}_{3}^{2}$ & 298 & 0.48 \\
\hline 7. & 18.77 & 10-Undecenoic acid, octyl ester & $\mathrm{C}_{19}^{18} \mathrm{H}_{36}^{34} \mathrm{O}_{2}^{3}$ & 296 & 1.62 \\
\hline 8. & 22.16 & Squalene & $\mathrm{C}_{30}^{19} \mathrm{H}_{50}^{36}$ & 410 & 0.52 \\
\hline 9. & 27.66 & Cis-Z- à-Bisabolene epoxide & $\mathrm{C}_{15}^{30} \mathrm{H}_{24}^{50} \mathrm{O}$ & 220 & 2.64 \\
\hline 10. & 28.00 & Cholestan-3-ol,2-methylene-,(3à,5à)- & $\mathrm{C}_{28}^{15} \mathrm{H}_{48}^{24} \mathrm{O}$ & 400 & 4.44 \\
\hline 11. & 28.87 & Vitamin A aldehyde & $\mathrm{C}^{28} \mathrm{H}_{28} \mathrm{O}$ & 284 & 2.28 \\
\hline 12. & 29.22 & Lumicolchicine & $\mathrm{C}_{22}^{20} \mathrm{H}_{25} \mathrm{O}_{6}$ & 399 & 10.22 \\
\hline 13. & 30.00 & Ethyl iso-allocholate & $\mathrm{C}_{26}^{22} \mathrm{H}_{48}^{23} \mathrm{O}_{5}$ & 436 & 7.89 \\
\hline
\end{tabular}

Table 5 : Phyto-components obtained through GC-MS analyzing isolated from methanolic tuber extracts of Gloriosa superba $\mathrm{L}$ (GA5-Vedaranyam) ecotype

\begin{tabular}{|c|c|c|c|c|c|}
\hline S.No. & RT & Name of the compound & Molecular formulae & MW & Peak area \% \\
\hline 1. & 9.49 & Lactose & $\mathrm{C}_{12} \mathrm{H}_{22} \mathrm{O}_{11}$ & 342 & 10.11 \\
\hline 2. & 11.01 & Undecanoic acid & $\mathrm{C}_{11}^{12} \mathrm{H}_{22}^{22} \mathrm{O}_{2}$ & 186 & 1.76 \\
\hline 3. & 12.05 & n-hexadecanoic acid & $\mathrm{C}_{16}^{11} \mathrm{H}_{32} \mathrm{O}_{2}^{2}$ & 256 & 29.81 \\
\hline 4. & 14.00 & 9,12-0ctadecadienoic acid $(Z, Z)$ - & $\mathrm{C}_{18}^{10} \mathrm{H}_{32}^{32} \mathrm{O}_{2}^{2}$ & 280 & 36.73 \\
\hline 5. & 16.66 & 10-Methyl-E-11-tridecen-1-ol propionate & $\mathrm{C}_{17}^{18} \mathrm{H}_{32}^{32} \mathrm{O}_{2}^{2}$ & 268 & 0.35 \\
\hline 6. & 17.97 & 2-Hydroxy-(Z) 9-pentadecenyl propanoate & $\mathrm{C}_{18} \mathrm{H}_{34} \mathrm{O}_{3}$ & 298 & 0.83 \\
\hline 7. & 18.79 & 10-Undecenoic acid, octyl ester & $\mathrm{C}_{19}^{16} \mathrm{H}_{36} \mathrm{O}_{2}$ & 296 & 1.73 \\
\hline 8. & 22.18 & Squalene & $\mathrm{C}_{30}^{19} \mathrm{H}_{50}^{36}$ & 410 & 0.59 \\
\hline 9. & 27.66 & Cis-Z- à -Bisabolene epoxide & $\mathrm{C}_{15}^{30} \mathrm{H}_{24}^{50} \mathrm{O}$ & 220 & 1.86 \\
\hline 10. & 28.01 & Cholestan-3-ol, 2-methylene-,(3à,5à)- & $\mathrm{C}_{28}^{15} \mathrm{H}_{48}^{24} \mathrm{O}$ & 400 & 3.21 \\
\hline 11. & 28.87 & Vitamin A aldehyde & $\mathrm{C}_{20}^{28} \mathrm{H}_{28}^{48} \mathrm{O}$ & 284 & 1.96 \\
\hline 12. & 29.24 & Lumicolchicine & $\mathrm{C}_{22}^{20} \mathrm{H}_{25}^{28} \mathrm{NO}_{6}$ & 399 & 9.83 \\
\hline 13. & 29.95 & Ethyl iso-allocholate & $\mathrm{C}_{26}^{22} \mathrm{H}_{44} \mathrm{O}_{5}{ }^{6}$ & 436 & 1.21 \\
\hline
\end{tabular}

acid, 9,12- Octadecadienoic acid (Z,Z), 10-Methyl-E-11Tridecen-1-ol propionate, 2-Hydroxy - (Z) 9-Pendadecenyl propanoate, 10- Undecenoic acid, Octyl ester, Squalene, CisZ-a-Bisabolene epoxide, Cholestan -3-ol, Vitamin A aldehyde, 2-Methylene-,(3à,5à)- Lumicolchicine and Ethyl iso-allocholate were reported (Table 5 and Figure 6).

The chemical compound Lactose was reported in Gloriosa superba L ecotypes of GA3, GA4 and GA5. 9,12-Octadecadienoic acid (Z,Z)-, n-hexadecanoic acid were reported in GAl, GA2, GA3, GA4 and GA5.Squalene was reported in GA1, GA4 and GA5 ecotypes.Vitamin A aldehyde found in GA4 and GA5 ecotypes. Lumicolchicine was present in ecotypes of GA2, GA3, GA4 and GA5 respectively. Ethyl allocholate is found in ecotypes of GA2, GA4 and GA5.

\section{DISCUSSION}

Our study reveals that Gloriosa superba L tuber contains various phyto-chemicals including alkaloids, steroids, triterpenoids, saponins and tannins. The results supported similar findings observed by Listsea glutinosa [18]. GC-MS analysis of drugs revealed that the presence of various phyto-chemicals, including alkaloids and other observed structured compounds in GC- MS analysis in our findings[19].

Medicinal plant derived phyto-chemicals are an important source of medicinal plants and for eco friendly applications [20]. Plant extracts and phyto-components are active against various diseases [21]. In the present investigation, methanol extracts of tuber Gloriosa superba L from five different ecotypes such 
as Sirumalai (GA1), Mulanoor(GA2), Thuraiyur (GA3), Konganapuram (GA4) and Vedaranyam (GA5) contain various phyto-components. The results are obtained in accordance with the previous reports.

The analysis indicates that domesticated plant of Gloriosa superba L morphologically as well as physiologically divergent from their ancestor wild ecotypes. The present investigation reveals a comparative analysis of the phyto-components of five different accessions of Gloriosa superba L from Tamil Nadu state, India by using GC-MS method. Most of the accessions possess higher phyto-components in the tuber of Gloriosa superba L.

\section{CONCLUSION}

The present investigation on the phyto-components of Gloriosa superba L accessions may be utilized as an application oriented tool to find out a new source of natural antioxidants, pharmaceutical applications in a quantitative and qualitative approach.

\section{ACKNOWLEDGEMENTS}

The authors are thankful to Indian Institute of Crop Processing Technology (IICPT), (Ministry of Food Processing Industries, Government of India, Tanjore), Tamil Nadu to carry out the work successfully.

\section{REFERENCES}

1. Srivastava UG, Chandra V. Gloriosa superba Linn. (Kalihari) - An important colchicines producing plant. J Res Indian Med. 1977; 10:92-5.

2. Prajapati ND, Purohit SS, Sharma AK, Kumar TA. A Handbook of medicinal plants. Agrobios Publishers (India) Jodhpur. 2003;221-234.

3. Asha V, Narmratu S, Aravind K. Phyto chemical investigation and thin layer chromatography of Asparagus racemosus (Family Asparagaceae) methanolic leanes extract. International Journal of Advanced Research in Pharmaceutical and Bioscience. 2013; 3(1):15-18.

4. Bose TK, Chowdhary K. Tropical garden plants in colour: A guide to tropical ornamental plants for garden and home. 1991.

5. Prince L, and Prabakaran P. Antifungal activity of medicinal plants against plant pathogenic fungus Colletrotrichum falcatum. Asian J.Plant Sci. Res. 2011; 1(1):84-87.

6. Baskar UC, Dash D, Mahapatra AK, Estimation of Colchicine in tubers of Gloriosa superba L, originated from different agroclimatic zones of Odisha, India, International Journal of Pharmacognosy and Phytochemical Research. 2012; 4(3):157-161

7. Gupta LM, Raina RC, Raina R, Gupta M. Colchicine content in Gloriosa superba L - J. Res. SKUAST-J. 2005; 4(2):238-241

8. Rizwan SA, Abdul rahman AG, Raisuddin A, Wajhu Q, and Suliman A. Analysis of inorganic and organic constituents of myrrh resin by GC-MS and ICP-MS: An emphasis on medicinal assets. Saudi Pharmaceutical Journal. 2017; 25:788-794.

9. Evans WC. Trease and Evan's Pharmacognosy. $14^{\text {th }}$ ed, UK: WB Sauvaders Company Limited. 1997; P.3.

10. Anbuselvi S, Jeyanthi Rebecca L, Sathish kumar M, Senthilvelan T. GC-MC study of phytochemicals in black gram using two different organic manures. Journal of Chemical and Pharmaceutical Research. 2012; 4(2):1246-1250

11. Makky EA, Mashitah M, Yus off, Ibrahim MM. Impact of Medicinal plants phyto-components against Antibiotic Resistant Bacteria. Journal of Chemical and Pharmaceutical Research. 2012; 4(1):881-893.

12. Kavitha R, Kamalakannan S, Deepa T, Elamathi R, Sridhar S, Suresh kumar J. In vitro antimicrobial activity and phytochemical Analysis of Indian Medicinal plant Couroupita guianensis. Journal of Chemical and Pharmaceutical Research. 2011; 3(6):115-121.

13. Meurer, Grimes B, Mcbeth DL, Hallihan B, Delph S. Antimicrobial activity in medicinal plants of the Scrophulariaceae and Acanthaceae. International Journal of Pharmacognosy. 1996; 34:243-248.

14. Koduru S, Grierson D.S, Afolayan A.J. Antimicrobial activity of Solanum aculeastrum. Pharmaceutical Biology. 2006; 44:283-286.

15. Shipra B, Kshipra D, Amla B, Asha S, Bharti M. Zingiber officinale chemical and physiochemical screening and evaluation of its antimicrobial activities. Journal of Chemical and Pharmaceutical Research. 2012; 4(1):360-364.

16. Kumaravel S, Praveenkumar P, and Vasuki, P. GC-MS study on microbial degradation of Lindane. International Journal of Applied Chemistry. 2010; 6(3):363-366.

17. Priya D, Rajaram K, Sureshkumar P. Phyto chemical studies and GC-MS analysis of Caralluma fimbriata Wall. International Journal of Pharmaceutical Research and Development. 2011; 3(10):105-110.

18. Mandal SC, Kumar, CKA, Majurnder A, Majumder R, and Maity BC Antibacterial activity of Listsea glutinosa bank. Fitoterapia. 2000; 71(4):439-441

19. Yang XX, Hu ZP, Duan W, Zhu YZ, Zhou S.F. Drug- herb interactions: eliminating toxicity with had drug design. Curr. Pharma Res. 2006: 12:4649-4664.

20. Venkatachalam MR, Jebasan A. Larvicidal activity of Hydrocotyl javanica Thump extract against Culex qunquefasciatus. J. Exp to Zool. India. 2010; 4:99-101.

21. Sun L, Dong H, GCo C, Qian J, Sun J, Ma L, Zhu C. Larvicidal activity of extracts of Ginkgo biloba exocarp for three different strains of Culex pipienspallens. J.Med. Entomol. 2006; 43:258-261 